\title{
COMPARATIVE EVALUATION OF CONTROL-ORIENTED ZONE TEMPERATURE PREDICTION MODELING STRATEGIES IN BUILDINGS*
}

\author{
Venkatesh Chinde, Jeffrey C Heylmun, Adam Kohl, Zhanhong Jiang, Soumik Sarkar, Atul Kelkar \\ lowa State University, Ames, IA 50011 \\ Emails: \{vchinde@iastate.edu, jheylmun@iastate.edu, adamkohl@iastate.edu, \\ zhjiang@iastate.edu, soumiks@iastate.edu, akelkar@iastate.edu\}
}

\begin{abstract}
Predictive modeling of zone environment plays a critical role in developing and deploying advanced performance monitoring and control strategies for energy usage minimization in buildings while maintaining occupant comfort. The task remains extremely challenging, as buildings are fundamentally complex systems with large uncertainties stemming from weather, occupants, and building dynamics. Over the past few years, purely data-driven various control-oriented modeling techniques have been proposed to address different requirements, such as prediction accuracy, flexibility, computation and memory complexity. In this context, this paper presents a comparative evaluation among representative methods of different classes of models, such as first principles driven (e.g., lumped parameter autoregressive models using simple physical relationships), datadriven (e.g., artificial neural networks, Gaussian processes) and hybrid (e.g., semi-parametric). Apart from quantitative metrics described above, various qualitative aspects such as cost of commissioning, robustness and adaptability are discussed as well. Real data from Iowa Energy Center's Energy Resource Station (ERS) test bed is used as the basis of evaluation presented here.
\end{abstract}

\section{Introduction}

Managing energy demand in an increasingly urban world and developing sustainable energy sources with minimal environmental impact are among the most challenging current technological problems. According to the U.S. Department of En-

${ }^{*}$ THE AUTHORS WOULD LIKE TO THANK IOWA ENERGY CENTER FOR SUPPORTING THIS WORK - OPPORTUNITY GRANT NO. OG-15-005.

${ }^{\dagger}$ Address all correspondence to this author. ergy, approximately $40 \%$ of the total energy usage in the U.S. today is consumed by the building sector (residential $22 \%$, commercial 18\%) whereas the balance is evenly distributed between industry and transportation sectors [1]. Therefore, energyefficient building technology is absolutely critical a fact that is reflected in the aggressive targets set by the U.S. government and other agencies: $20 \%$ reduction in annual energy use in commercial buildings by 2020; $50 \%$ reduction in energy consumption in the building sector by 2030. Similar targets are in place for the EU and other parts of the world as well [2]. Therefore, the Department of Energy (DOE), the International Energy Agency (IEA), the Intergovernmental Panel on Climate Change (IPCC) and other agencies have declared a need for commercial buildings to become 70 - $80 \%$ more energy efficient. Efficient buildings will not only lead to energy savings but will also lower the greenhouse gas emissions and help fight climate change and reduce costs.

Traditional energy-saving technologies include efficient Heating, Ventilation and Air Conditioning (HVAC), lighting systems, smart architectural design (facades, windows), and local energy harvesting (solar, heat recovery) processes. However, with the advent of advanced sensing and computational resources, information technologies such as integrated performance monitoring, health management, and optimal supervisory control are expected to have significant impact in this sector for both new and existing buildings. The large potential economic impact of advanced technologies underlying modern Building Management Systems (BMS) have led to increased efforts focused on developing, designing, and implementing model-based control and monitoring technologies for building HVAC sys-
Copyright (C) 2015 by ASME 
tems. Today's state-of-the-art building management systems (BMSs) mostly use rule-based decentralized decision systems for monitoring, scheduling and controlling large commercial buildings [3]. However, achieving optimality requires dedicated and time-consuming commissioning and tuning effort in each building. Moreover, they lack in robustness and adaptability to environmental/usage uncertainties. On the other end of the spectrum lies highly optimal, centralized supervisory control solutions (e.g., MPC based [4-6], [7], [8]) that have adaptive and predictive capabilities. However, such schemes can be prohibitive due to modeling/computational complexity and commissioning cost. Distributed optimization based control strategies $[9,10]$ can potentially alleviate such issues by treating different HVAC subsystems as different entities with individual performance goals. However, a completely decentralized solution may suffer from significant sub-optimality due to conflicting nature of objectives and constraints.

Irrespective of control scheme choice, control-oriented HVAC modeling remains indispensable in order to realize energy efficiency. Among various HVAC modeling aspects in a building, thermal modeling of building zones is one of the most important ones as maintaining comfort in zones is the primary task of a building HVAC control system. In general, this is a technically challenging issue due to various system heterogeneity as well as unstructured uncertainties such as occupant behavior and direct solar load. Among different types of such zone models, although the physics-based approaches capture relevant heat transfer characteristics, calibration and adaptation of those models to represent the real condition may be difficult. On the other hand, purely data-driven techniques may succumb to artificial correlations in data and overfitting issues. Furthermore, need for meticulous calibration and tuning increases cost of commissioning which is typically a key constraint in the building sector. Therefore, automated model learning and adaptation capability is extremely useful [11]. Note, along with prediction, quantifying model confidence (or uncertainty) may be critical in order to enhance robustness of controllers.

\section{Brief Descriptions of Modeling Schemes}

Among various control-oriented zone temperature modeling approaches that use physics-based and first principle knowledge, R-C networks [12], [13], [14] and Autoregressive models [5], [15] have been quite popular. However, as this paper focuses on modeling single zones separated by near adiabatic walls (as found in ERS test bed [16]), Autoregressive models such as ARX and ARMAX models are chosen for evaluation. Among purely data-driven tools, this paper uses Artificial Neural Networks [17-19] which have been the most popular. However, Gaussian Processes can also be quite effective as it inherently quantifies model confidence. Although the technique has mostly been used for building level energy usage prediction [20], this pa-

per explores its applicability for zone temperature modeling. Hybrid techniques such as semi-parametric [21], [22] modeling has been used where the parametric component uses physics based knowledge (lumped parameter model of zone-level heat transfer) and the nonparametric component helps capture the uncertainties from measured data. This reduces difficulties of searching appropriate models to describe zone specific behaviors. Brief description of each of the modeling scheme is presented below:

Autoregressive models with Physics-based kno
embedding: These models are composed of an aut
sive part (in ARX) and/or a moving average part
MAX) and exogenous disturbances (d(n)). The me
incorporated in the autoregressive part and moving
models are so called random shock terms. The exo
variables are heating load due to occupancy and sola
tion. The generic (discrete-time) model is described
\[ T(n+1)=\sum_{i=0}^{n_{a}} a_{i} T(n-i)+\sum_{j=0}^{n_{b}} b_{j} T_{o a}(n-j) \]
$+\operatorname{cim}(n)\left(T_{s}(n)-T(n)\right)+d(n)$

where $T(n), T_{s}(n)$ and $T_{o a}(n)$ denote zone temperature, supply air temperature and outside air temperature respectively as functions of time; $a_{i}, b_{j}, \mathrm{c}$ are constants and $\dot{m}(n)$ is the mass flow rate; $n_{a}, n_{b}$ denote the auto regressive orders for zone temperature and outside air temperature. Note, for an ARX model $n_{b}=0$. A direct comparison of the ability of both ARX and ARMAX models to predict zone temperature is studied in [23], [24].

Artificial Neural Networks (ANN): These are purely datadriven black box models and have been extensively used as nonlinear input-output maps in various problems. The most common neural network structure is the Multi-LayerPerceptron (MLP) trained using the back-propagation algorithm. The neural networks are trained based on standard nonlinear autoregressive models with exogenous inputs (NARX) [18]. The back-propagation algorithm is an iterative gradient based algorithm designed to minimize the mean square error between the actual and desired output. In [18] ANNs are used to predict the future temperature in a single zone, multiple zones, and the effects of thermal coupling. The perceptron computes a single output from multiple real-valued inputs by forming a linear combination according to its input weights and then possibly putting the output through some nonlinear activation function. Mathe- 
matically this can be expressed as

$$
y=\sum_{j=1}^{n_{h}} W_{j} f\left(\sum_{i=1}^{n_{u}} w_{i j} x_{i}+b_{i}\right)+B_{1}
$$

where $W_{i}$ and $w_{i j}$ are the weights of the ANN to be estimated for the output and hidden layer respectively, and also $b_{i}$, $B_{1}$ are the weights for the bias connection with the hidden and output layers respectively. The hidden layer uses a logistic sigmoid function $f$ as the activation function and the weights and bias of that layer were initialized using the Nguyen-Widrow method to ensure a more consistent result and the inputs of the model were normalized and prepared with a time delay of two to be consistent with the other models. Lastly, the training is halted as soon as the target mean squared error reached in order to keep the network from becoming over-trained.

Gaussian Process (GP): GP [25], [26] is a powerful and flexible uncertainty quantification and data modeling technique that enables the construction of complex models without the need of specifying algebraic relationships between variables by assigning a structure for the covariance matrix of input variables to compute predictions of output variables. It is derived from the Bayesian framework which naturally provides predictive probability distributions. As a result, GP models can capture complex nonlinear relationships between multiple input and output variables and can provide mean predictions and associated uncertainty levels. Hence, effects of uncertainties in sensor data (due to weather, occupancy and solar radiation) can be captured using GP models. The maximum likelihood approach is adopted to find the optimal set of hyper-parameters [27] associated with the mean and covariance functions.

Semiparametric: Recently [21], this statistical approach has been used in the context of building systems for identifying models which are suitable for control design. Zone model is influenced by both parametric and non-parametric (heating load) components. Therefore, it can be modeled using semi-parametric regression. The accuracy of zone model is largely affected by the heating load due to occupancy, equipment and solar impact. An example dynamic model of a zone is given by partial linear model described below, which is similar to the (1) and the key difference is the presence of non-parametric part.

$$
\begin{array}{r}
T(n+1)=\sum_{i=0}^{n_{a}} a_{i} T(n-i)+\sum_{j=0}^{n_{b}} b_{j} T_{o a}(n-j)+q(n) \\
+c \dot{m}(n)\left(T_{s}(n)-T(n)\right)+\varepsilon(n)
\end{array}
$$

where $q(n)$ is the heating load that is a time varying quantity and $\varepsilon(n)$ is assumed to be IID with zero mean and constant variance that is conditionally independent on the variables of model. The parametric and non-parametric components are estimated using ordinary least squares and kernel regression method. For more details see [21].

\section{Performance comparison using Physical Building Test bed}

To illustrate and compare the effectiveness of different methods in terms of various metrics, we have used real data form the Iowa Energy Center's Energy Resource Station (ERS) test bed. The test bed consists of eight zones distributed in four directions, East, South, West, and North, respectively. All the test zones within the ERS are intended to simulate a typical office space and each zone has approximately 266 sq.ft of floor space. All zone and air handling system controls uses a modern programmable commercial-grade Direct Digital Control (DDC) system - Distech Controls' EC-NetAX ${ }^{\mathrm{TM}}$ Building Management System. The DDC system controls all air distribution equipment. The DDC system is fully equipped with instrumentations and sensors required for various building energy efficiency related research. Overall there are over 1,200 monitoring and control points with 600 of them the data are collected every minute. Note that the zones in north direction are also interior as they have no windows and the zones in other directions have exterior windows, hence they are perimeter zones. In each direction, two constructed zones marked $\mathrm{A}$ and $\mathrm{B}$ have identical exposures yielding identical external thermal loads and may have identical internal thermal loads thereby allowing simultaneous, side-byside comparison testing of many types of HVAC systems and control schemes. For example, in B zones there are fan coil units in addition to variable air volume (VAV) boxes to heat the zone if necessary. However, the fan coil units were always off during data collection time windows such that conditions for zones A and $\mathrm{B}$ are completely identical. The schematic diagram is shown in Fig. 1. Some zones are connected with other offices and spaces. However, the effects caused by the connection with other offices and spaces were ignored due to strict separation steps taken during data collection times. Also, the test zones have some other equipment, such as computers and lighting facilities. They were always off such that we need not consider their effect either. Evidently, interior zones do not receive no solar radiation due to the absence of windows. On the other hand, zones located in other three directions receive solar radiation during the day time. In each zone, there are several sensors installed to measure variables, e.g., zone temperature, air volumetric flow rate, and discharge air temperature from VAV boxes which are used to train the different models described in the modeling section.

A detailed performance comparison of the different methods 


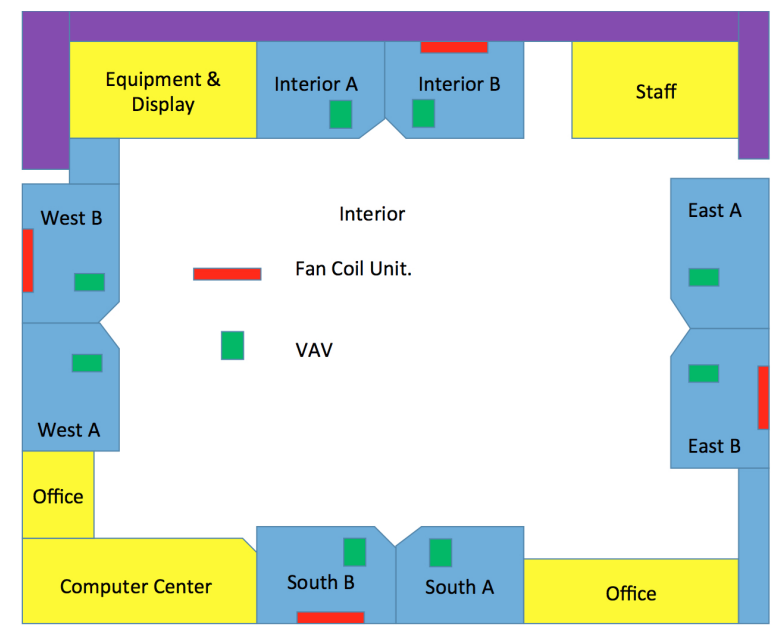

FIGURE 1. Schematics of ERS Test bed

on various aspects such as accuracy, computation time, memory requirement, robustness and cost of commissioning which are necessary for having a reliable model in terms of control design and analysis is shown in Table 1 and Table 2. Most quantitative results are based on training data consisting of 720 samples picked from a window of two weeks (from April 2011) in order to take into account different zone temperature characteristics and six days of validation data (from April 2011) unless otherwise mentioned. Also, the comparative analysis presented in the tables primarily uses one interior zone behavior over the six validation days. The models are simulated on a $3.30 \mathrm{GHz}$ Intel(R) $\mathrm{Xeon}(\mathrm{R})$ processor in the MATLAB R2014a environment. The model predictions are done in near-real time, the sampling rate of prediction is $1 \mathrm{~min}$ and the information used are the initial temperature values and the inputs. Typically, different zones in a building has different characteristics based on the solar load and other uncertainties. Fig. 2 shows zone temperature prediction performances of all the modeling schemes on the consecutive six day testing period. In the sequel, we focus on a particular testing day and evaluate each method separately. In order to show the effectiveness of these models for different scenarios, we have also considered a zone (east) which is affected largely by uncertain solar load. Similarly, a time window which is at the end of the season (during May 2011, end of Spring) is also chosen for evaluation of the modeling schemes under slight seasonal variations.

ARX/ARMAX: ARX/ARMAX are linear parametric models that use physics based relationships, such as heat transfer from the HVAC unit (VAV) significantly in order to predict zone temperature. Presence of control variables such as air flow rate and discharge air temperature enables us to use this type of models for controller design purpose. Furthermore, the weather characteristics can be included in discrete form and the dynamic

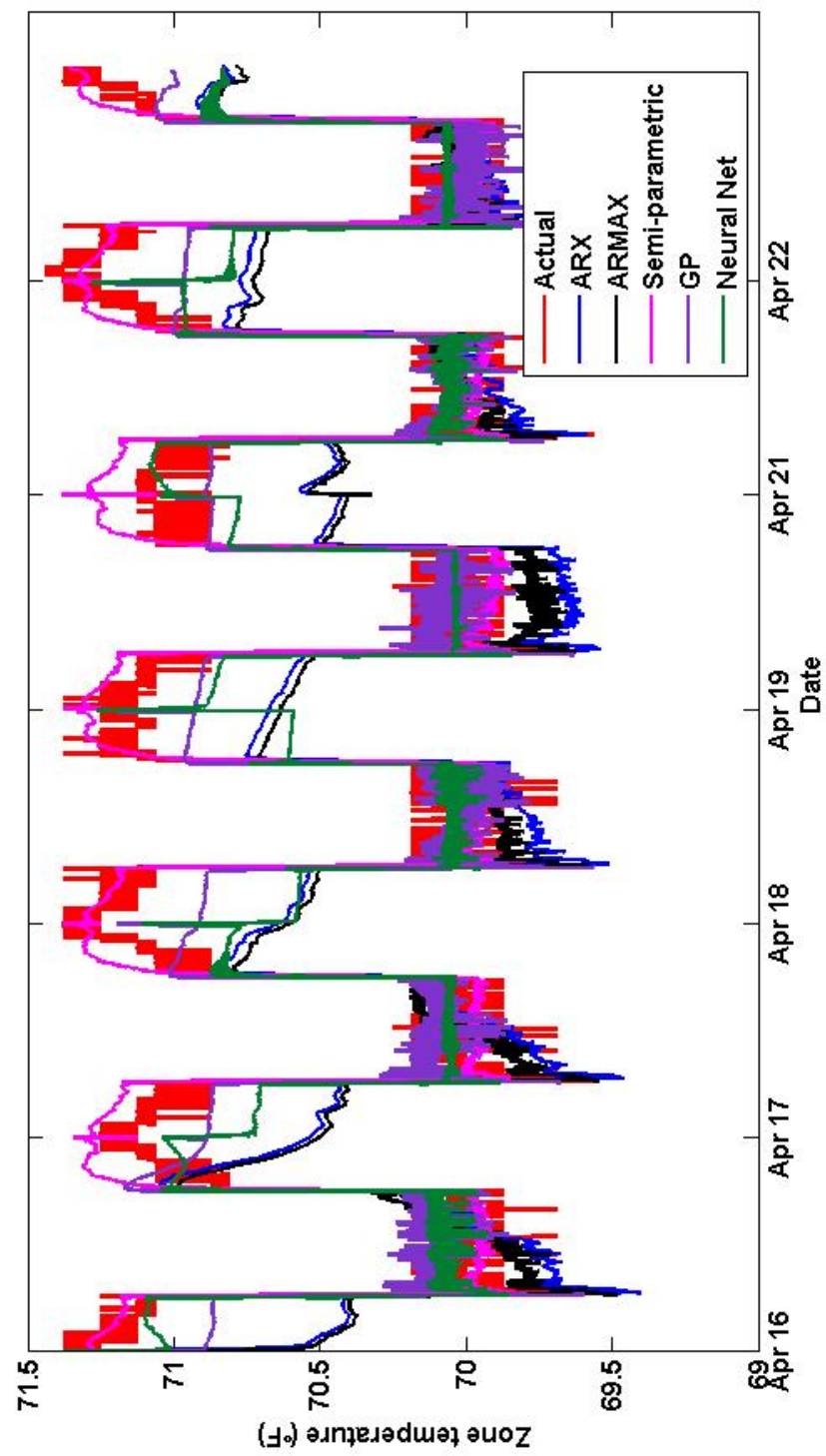

FIGURE 2. Zone temperature prediction for six validation days using different methods 
zone model can be obtained by means of linear autoregressive techniques relating input and output of the system. The results using both ARX and ARMAX methods are shown in Fig.3 and Fig.4 respectively. As the plots show, both methods predict

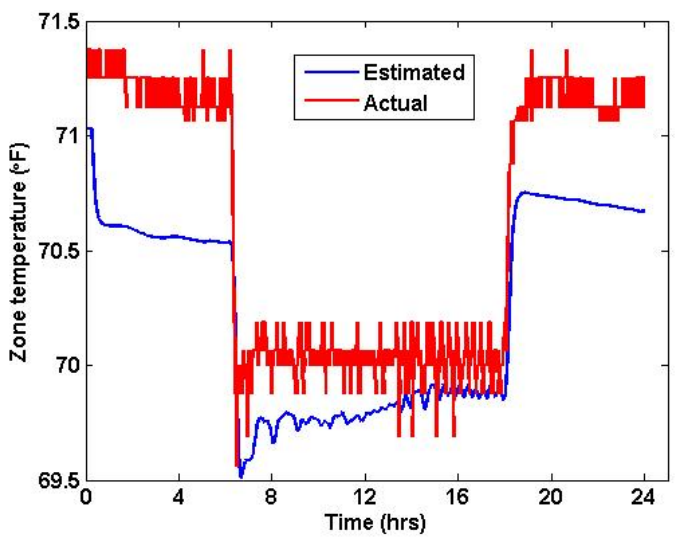

FIGURE 3. Zone temperature prediction ARX model

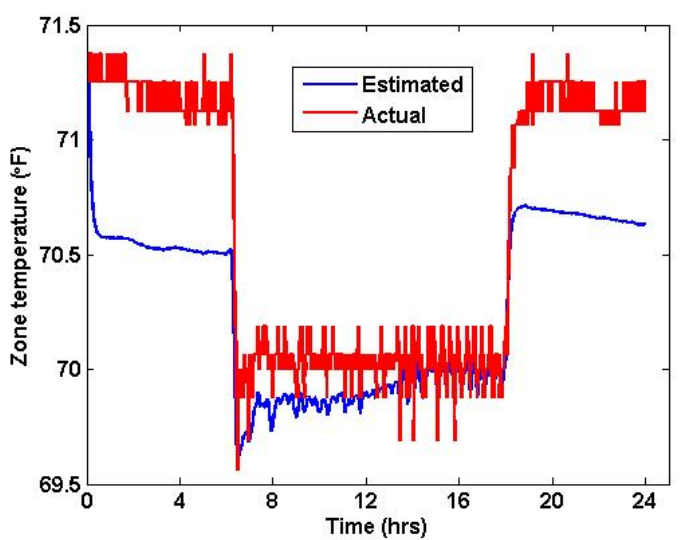

FIGURE 4. Zone temperature prediction using ARMAX model

zone temperature conditions reasonably well during the occupied hours (when the HVAC is active) with a maximum error of about $1^{\circ} \mathrm{F}$ and suffer during the time when the HVAC is off. This can be explained by the fact that since these models use physics based relationships, therefore, without active heat transfer to the zone, they do not perform that well as they are unlikely to capture the heating load dynamics effectively. ARMAX is slightly more effective compared to ARX in predicting zone temperature with relatively smaller prediction errors.
On a more qualitative note, several observations related to the usability of the models can also be made in terms of ease and cost of implementation, robustness and time complexity. These models are easily implemented (due to the small number of hyper-parameters) and use the available data very efficiently in order to accurately update the models. The training time is quick (less than a second with 720 samples of training data) since the model complexity is quite low and they use a great deal of prior knowledge based on physics.

Artificial Neural Network: The multilayered neural network is probably one of the most frequently used types of ANN structure in practical applications. The architecture of the network includes an input layer, one or more hidden layers, and an output layer. Fig. 5 shows the prediction capability of the ANN

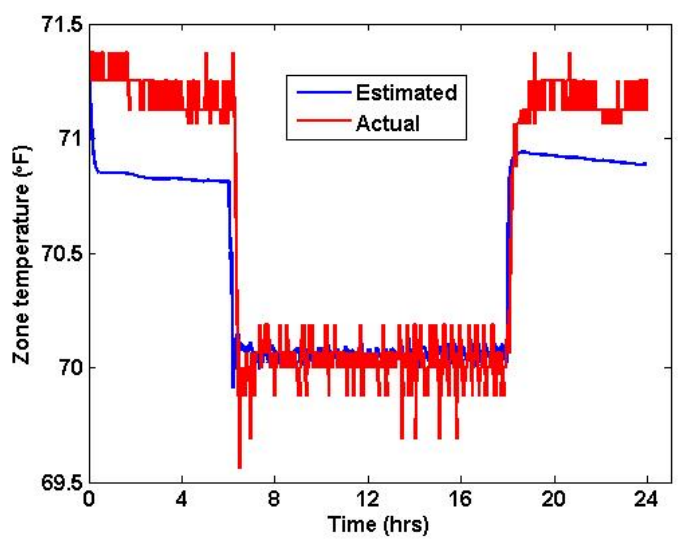

FIGURE 5. Zone temperature prediction using Artificial Neural Network model

model, the validation day was very similar to that of training days. Due to similar characteristics of the Training and Validation days, the weights and biases of the ANN are properly trained to give better performance. The prediction accuracy is slightly better compared to ARX/ARMAX models and especially during the occupied hours.

Gaussian Process: GP is one of the non-parametric machine learning approaches that avoids the model specification problem and learns directly from the available data. For Gaussian process simulation, we have used a squared exponential covariance function [27] and a composite mean function which amounts to a total of ten hyperparameters and all with zero initial conditions. The output of validated GP model is shown in Fig.6. Mean prediction along with model confidence bound (two sigma) are presented. Note, localized confidence bounds in this 
case signify availability of training data in a particular region. Hence, in the case there is lack of training data for a certain region in the data space, GP automatically detects it with a larger variance.

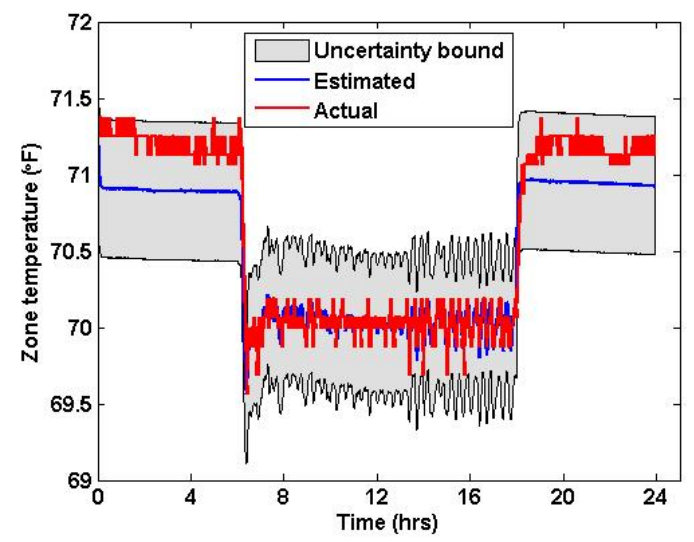

FIGURE 6. Zone temperature prediction using Gaussian process model

We can see that the zone temperature lies within the $95 \%$ measure ( 2 sigma bound) of confidence interval. The model performs significantly better in both occupied and unoccupied hours compared to other physics and data driven models, the error is less than $0.5^{\circ} \mathrm{F}$ during unoccupied hours and even less during occupied hours.

The primary disadvantage associated with the GP model is the high computational burden, associated with learning of hyper-parameters. As the training data size and the number of hyper-parameters increases, the training time increases and requires a large amount of training data to cover the entire data space. Therefore, GP-based modeling can be a good choice for initial off-line modeling, but may not be ideal for online adaptation purposes. The results can be potentially improved by using prior knowledge of hyper-parameters and also by incorporating local model (linear/nonlinear) into GP.

Semiparametric: In general, zone temperature model is a blend of both parametric and non-parametric components. The primary non-parametric component in zone temperature modeling is the heating load due to solar radiation, equipment and occupancy. In this model, the heating load is identified only using temperature measurements [21], which makes the model simpler and is readily used for controller design purpose. The average heating load during the training phase is used for validation purpose.Temperature dynamics can be accurately predicted if the non-parametric component is accurate enough. As shown in Fig.7, semiparametric model performs well both in occupied and unoccupied hours compared to other methods in terms of accuracy. Although the zone is an interior zone, where the heating load is comparatively smaller this method performs the best. The advantage of this model is it doesn't require any additional

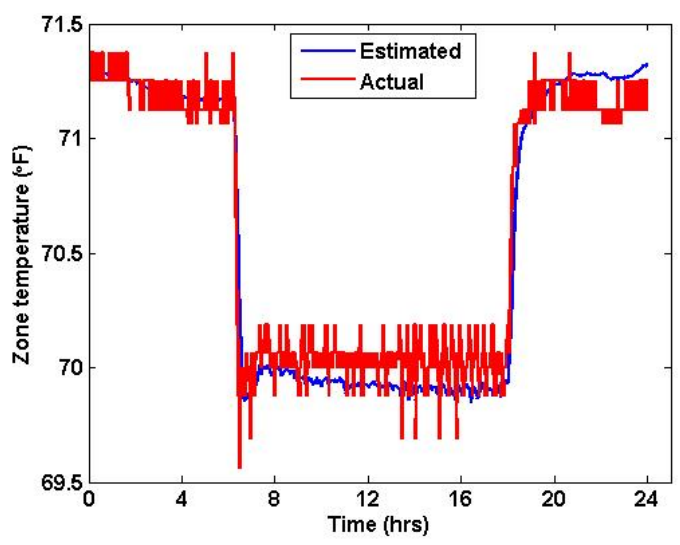

FIGURE 7. Zone temperature prediction using Semi-parametric model

sensors to measure heating load and it is used for quantifying energy consumption in terms of several factors such as the outside air temperature (OAT) and other factors [28]. This method has been successfully tested on various test beds [21]. This model has been effectively used for several examples with increased complexity, several nonlinearities and input-output noise. These models can be easily deployed and has the same memory complexity as compared to ARX/ARMAX models. In terms of robustness, they are quite robust in predicting temperature in the same season which the training data belongs to.

\section{Impact of worst-case uncertainty:}

In order to evaluate the models for rather difficult (or, worstcase uncertainty) scenarios, we consider a perimeter zone (east), which shares a large window with the outdoors and largely affected by the solar load. Furthermore, a daily temperature profile is chosen that has a significantly different pattern compared to the training patterns. Fig.8 shows comparison of different methods in predicting the zone temperature under that condition. The result with the ARX/ARMAX model captures the trend in the initial hours of the day and also the abrupt change in the morning hours when the heating load starts to increase due to solar load and typically the error is of the order of maximum $3^{\circ} \mathrm{F}$. The performance of purely data-driven models (ANN and GP) degrades significantly compared to other methods in terms of accuracy. As typically in the morning hours, heating load becomes large due to 
which it captures the jump during the initial occupied hours and later it tries to capture the nearly constant dynamics and the comparison in terms of different metrics is listed in Table. 1. For the

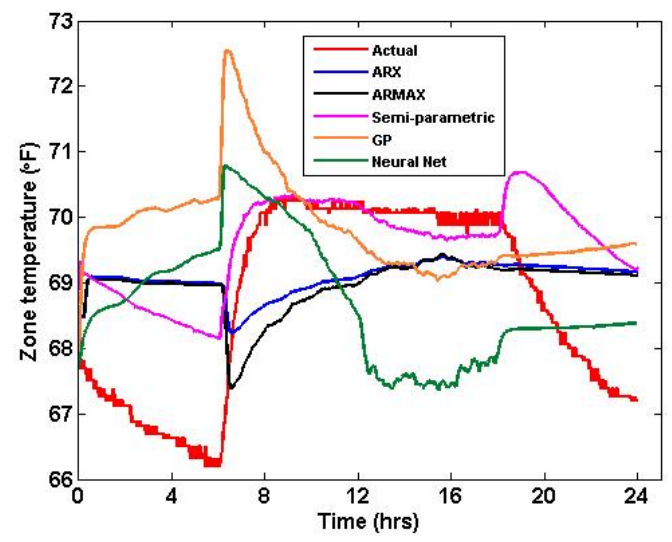

FIGURE 8. Zone temperature prediction accuracy using different methods for perimeter zone

Semiparametric model, the average of all the training days heating load is used to validate on different days. In Fig.8, the model performs well in occupied hours compared to all the other methods, which is typically when the heating load is large. During the rest of the day, there is a error less than $3^{\circ} \mathrm{F}$ due to the fact that the test day shows a quite different characteristics in terms of small heating load during the unoccupied hours. Adding constraints on the coefficients or using regularization, the coefficients can be tuned accordingly to physically represent the system and improved accuracy can be obtained. Errors for ARX/ARMAX are around $3^{\circ} \mathrm{F}$. The drawbacks associated with these models are that they are not good at estimating the heating load on the zone and provide crude approximations of the actual system dynamics. However, they are better in terms of performance compared to other methods, since these methods are driven by physical laws.

\section{Analysis of an end of season day:}

In order to evaluate the model performances under slight seasonal variations and to show the robustness of modeling schemes, we have considered a day towards the end of the season (May 30th 2011, end of spring) for the interior zone. The result is shown in Fig.9 and it can be seen that the GP model is able to capture the abrupt changes in the initial occupied hours and the associated trend during that period, the performance degrades during the period when the HVAC is off. Note that there is no solar radiation and the change in the temperature is only due to outside air which is significantly different during the test day compared to that during the training days. Using ANN, the performance is better compared to all the other methods in the occupied hours and the error is large during rest since the AHU is off during those times and largely depends on the outside air temperature. The performance degradation of ANN, GP is because of change in data distribution due to seasonal variation and this shows the lack of extrapolation ability of purely data-driven methods. The

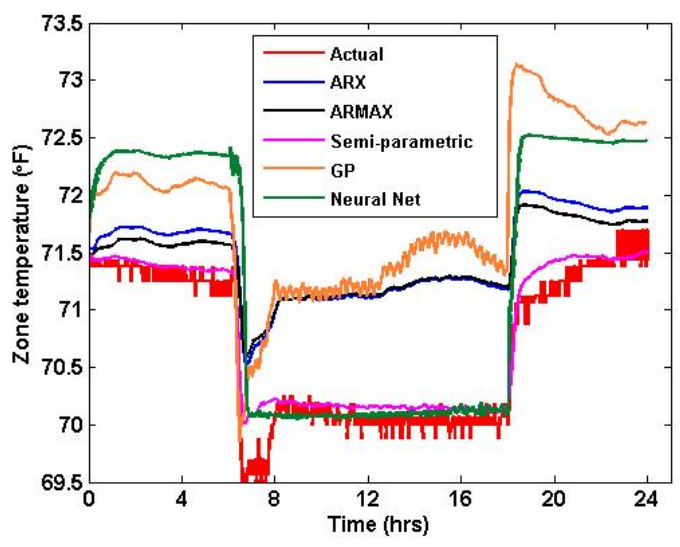

FIGURE 9. Zone temperature prediction accuracy using different methods for end of season day

results using Semiparametric model are better compared to other methods in terms of accuracy. Since, it is tested on the same season and the estimated heating load approximately matches with the actual heating load and this model can become worse compared to others when tested on other season days, the disadvantage of this model is that it is highly dependent on heating load and the results can be worse, if the temperature characteristics are highly different from that of training data. For ARX/ARMAX, the error is greater with the maximum error being less that $1.5^{\circ} \mathrm{F}$. This suggests, that such a model does not have to be re-trained as often as some other models would require. ARX/ARMAX and Semiparametric perform better compared to purely data-driven as they have slightly better extrapolation capability due to the involvement of physics-based relationships.

\section{Analysis of short-term prediction capabilities:}

It is evident that the performance of the models can be improved by updating the estimated temperature to actual temperature after certain fast time windows (15 minutes). This can be quite feasible as the supervisory control schemes typically work in a slower time scale and often allow update of initial conditions periodically (such as in receding horizon schemes). For example, we have considered the worst-case uncertainty data and the performance of ARX using 15 minute update after smoothing is shown in Fig.10. As expected, error is greatly reduced by updat- 


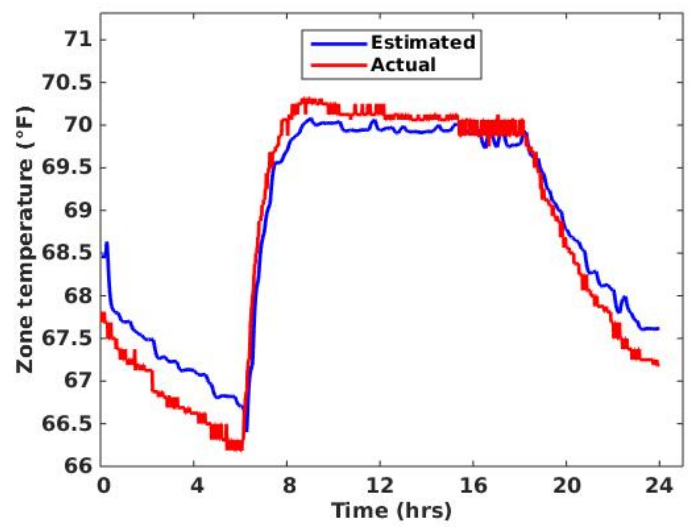

FIGURE 10. Zone temperature prediction accuracy using ARX for 15 min update after smoothing

ing the temperature to actual values. The maximum error goes down to $1^{\circ} \mathrm{F}$ as shown in Fig. 10 compared to $3^{\circ} \mathrm{F}$ in Fig. (8). The improved performance using the update for different methods is shown in Table.1.

\section{Summary and Conclusion}

In this paper, control oriented zone modeling techniques are reviewed and compared. Three main classes are identified which include first principle based, purely data-driven and hybrid methods. Data driven methods completely rely on the measurement data of the input and output variables and leaned functions to approximate the behavior of the system as closely as possible. These models include well established techniques such as Artificial Neural Networks and Gaussian Process. Another classification of models, which are physics based (ARX,ARMAX) they completely rely on the knowledge and the physical laws governing this process. The models built using physics based rules represent the system more closely and have better extrapolation capabilities. Purely, data-driven methods typically need comparatively larger amount of data to train the models. Another modeling technique which is a blend of physics based and data driven models. Hybrid models use physical laws to define the parametric part of the models and use measured data to find the function approximation of the nonparametric parts. For parameter identification, several optimization techniques such as least squares, gradient descent etc., can be used. Semi parametric model comes under this category.

The comparison of performance in terms of different metrics (qualitative and quantitative) for different classes of models used in the literature are presented. GP performs well compared to other methods in terms of accuracy. At the same time, it provides uncertainty bound (or model confidence) around the prediction. However, it suffers from larger training and validation time com- pared to ARX/ARMAX and Semiparametric models due to its iterative nature and similar time complexity can be observed for ANN, therefore they may not be ideal for fast online adaption purposes. ANN performs reasonably well and it shows a bias error that can be attributed to the overfitting issue. ANN, GP can perform well for off-line studies given enough data available for training purpose. ARX/ARMAX are easily implemented with the data available compared to other methods since they obey physical laws and errors can be reduced by applying the periodic reinitialization with actual measured values. Semiparametric performs the best compared to other methods when the training and validation days are in the same season due to the heating load approximation being accurate enough and can be used for online adaption. It was pretty robust too in all the weird conditions.

Based on the above discussion, many crucial advantages, disadvantages and insights are explored in this paper that can be significant for the building controls community and they can be used as guidelines for choosing modeling scheme in an appropriate manner for various applications.

\section{REFERENCES}

[1] EPA, 2008. "Report on environment, final report epa/600/r07/045f.".

[2] EUCOM, 2013. "Report from the commission to the european parliament and the council, brussels, $\operatorname{com}(2013) 225$ ".

[3] Tom, S., June 2008. "Managing energy and comfort". ASHRAE Journal.

[4] Bengea, S., Kelman, A., Borrelli, F., Taylor, R., and Narayanan, S., 2014. "Implementation of model predictive control for an hvac system in mid-size commercial building”. Journal of HVAC \& R Research, 20, pp. 121-135.

[5] Adetola, V., Bengea, S., Borrelli, F., Kang, K., Kelman, A., Leonardi, F., Li, P., Lovett, T., Sarkar, S., and Vichik, S., 2014. "Fault-tolerant optimal control of a large-size, commercial building heating, ventilation and air conditioning system". 3rd International High Performance Buildings Conference at Purdue.

[6] Bengea, S., Li, P., Sarkar, S., Vichik, S., Adetola, V., Kang, K., Lovett, T., and Leonardi, F., 2015. "Fault-tolerant optimal control of a building heating, ventilation, and air conditioning system". To appear in Science and Technology for the Built Environment (formerly HVAC\&R Research).

[7] Privara, S., Váňa, Z., Žáčeková, E., and Cigler, J., 2012. "Building modeling: Selection of the most appropriate model for predictive control". Energy and Buildings, 55, pp. 341-350.

[8] Mukherjee, S., Mishra, S., and Wen, J. T., 2012. "Building temperature control: A passivity-based approach.". In CDC, pp. 6902-6907.

[9] Elliott, M., and Rasmussen, B. P., 2015. "Optimal setpoints 

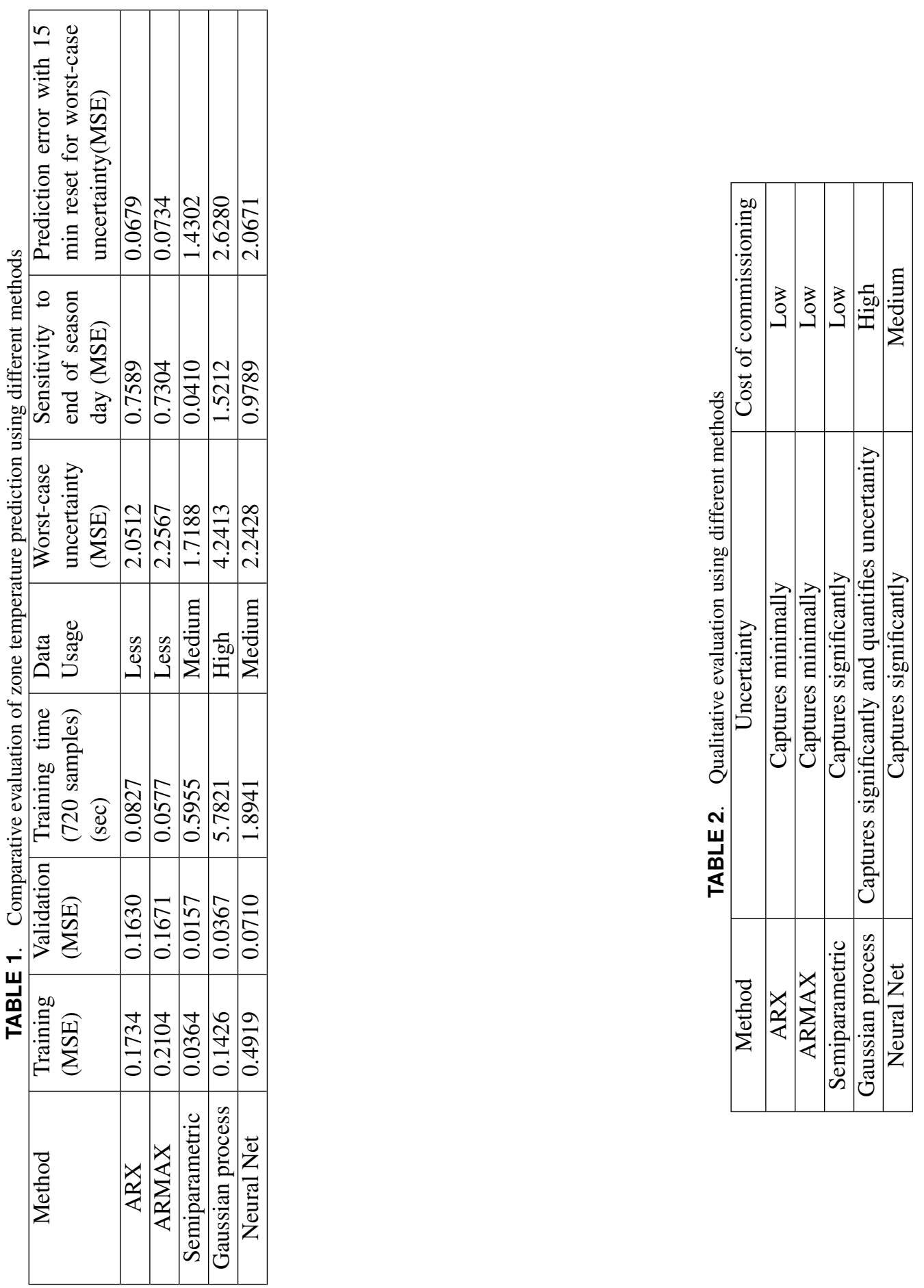
for hvac systems via iterative cooperative neighbor communication". Journal of Dynamic Systems, Measurement and Control, 137.

[10] Jalal, R. E., and Rasmussen, B. P., 2014. "Distributed model predictive control for networks with limited control communication". in the Proceedings of American Control Conference, Portland, OR.

[11] Chintala, R., Bay, C., and Rasmussen, B., 2014. "Automated modeling of building hvac systems for mpc". ASME Dynamic Systems and Control Conference, San Antonio, $T X$.

[12] Deng, K., Barooah, P., Mehta, P. G., and Meyn, S. P., July 2010. "Building thermal model redunction via aggregation of states". in Proceedings of the American Control Conference, Baltimore, $M D$.

[13] Goyal, S., Liao, C., and Barooah, P., 2011. "Identification of multi-zone building thermal interaction model from data". In Decision and Control and European Control Conference (CDC-ECC), 2011 50th IEEE Conference on, IEEE, pp. 181-186.

[14] Bacher, P., and Madsen, H., 2011. "Identifying suitable models for the heat dynamics of buildings". Energy and Buildings, 43(7), pp. 1511-1522.

[15] Wu, S., and Sun, J.-Q., 2012. "A physics-based linear parametric model of room temperature in office buildings". Building and Environment, 50, pp. 1-9.

[16] IEC, 2010. "Energy resource station (ers) technical description". Iowa Energy Center Technical Report.

[17] Kalogirou, S. A., and Bojic, M., 2000. "Artificial neural networks for the prediction of the energy consumption of a passive solar building". Energy, 25, pp. 479-491.

[18] Huang, H., Chen, L., Mohammadzheri, M., Hu, E., and Chen, M., June 2013. "Multi-zone temperature prediction in a commercial building using artificial neural network model". in the Proceedings of Control and Automation (ICCA), IEEE International Conference on.

[19] Huang, H., Chen, L., Mohammadzaheri, M., and Hu, E.,
2012. "A new zone temperature predictive modeling for energy saving in buildings". Procedia Engineering, 49, pp. 142-151.

[20] Srivastav, A., Tewari, A., Dong, B., Sarkar, S., and Gorbounov, M., 2014. "Localized uncertainty quantification for baseline building energy modeling, automated diagnostics for facility equipment, systems, and whole buildings, fairmont press".

[21] Aswani, A., Master, N., Taneja, J., Smith, V., Krioukov, A., Culler, D., and Tomlin, C., June 2012. "Identifying models of hvac systems using semiparametric regression". in the Proceedings of American Control Conference.

[22] Aswani, A., Master, N., Taneja, J., Krioukov, A., Culler, D., and Tomlin, C., 2012. "Energy-efficient building hvac control using hybrid system lbmpc". arXiv preprint arXiv:1204.4717.

[23] Ríos-Moreno, G. J., Trejo-Perea, M., Castaneda-Miranda, R., Hernández-Guzmán, V. M., and Herrera-Ruiz, G., 2007. "Modelling temperature in intelligent buildings by means of autoregressive models". Automation in construction, 16(5), pp. 713-722.

[24] Afram, A., and Janabi-Sharifi, F., 2014. "Review of modeling methods for hvac systems". Applied Thermal Engineering, 67(1), pp. 507-519.

[25] Heo, Y., and Zavala, V. M., 2012. "Gaussian process modeling for measurement and verification of building energy savings". Energy and Buildings(53), pp. 7-18.

[26] Rasmussen, C. E., 2006. "Gaussian processes for machine learning".

[27] Rasmussen, C. E., and Nickisch, H., 2010. "Gaussian processes for machine learning (gpml) toolbox". The Journal of Machine Learning Research, 11, pp. 3011-3015.

[28] Aswani, A., Master, N., Taneja, J., Krioukov, A., Culler, D., and Tomlin, C., 2012. "Quantitative methods for comparing different hvac control schemes". In Performance Evaluation Methodologies and Tools (VALUETOOLS), 2012 6th International Conference on, IEEE, pp. 326-332. 